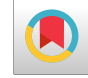

\title{
Psychopathology in a Patient with Lujan-Fryns Syndrome: A Case Report
}

\author{
Forouzan Elyasi (iD) ${ }^{1,}$, Arghavan Fariborzifar ${ }^{2}$ and Zahra Kashi ${ }^{3}$ \\ ${ }^{1}$ Department of Psychiatry, Psychiatry and Behavioral Sciences Research Center, Addiction Institute, School of Medicine, Mazandaran University of Medical Sciences, Sari, Iran \\ ${ }^{2}$ Research Student Committee, School of Medicine, Mazandaran University of Medical Sciences, Sari, Iran \\ ${ }^{3}$ Diabetic Research Center, School of Medicine, Mazandaran University of Medical Sciences, Sari, Iran \\ "Corresponding author: Consultation-Liaison Psychiatry Ward, Imam Khomeini General Hospital, Razi Ave., Postal Code: 48157-33971, Sari, Iran. Tel: +98-1133370885, Fax: \\ +98-1133363754, Emails: forouzan.el@gmail.com; f.elyasi@mazums.ac.ir
}

Received 2017 November 16; Revised 2019 August 10; Accepted 2019 August 31.

\begin{abstract}
Introduction: Lujan-Fryns syndrome (LFS) is an X-linked disorder characterized by varying degrees of symptoms, including mental retardation, Marfanoid habitus, facial deformities, hyper nasal speech, and psychopathology and related behavioral abnormalities. The prevalence and full spectrum of LFS's clinical symptoms remain unknown, but the disease is reportedly caused by at least one mutation in the mediator complex subunit 12 (MED12) gene.

Case Presentation: This study reports the case of an 18-year-old male with moderate mental disability, a height exceeding the 97th percentile, marfanoid characteristics, obvious craniofacial appearance, and psychiatric and behavioral disorders. Adults with LFS are usually tall, but their heights still fall within the normal range.

Conclusions: The higher-than-normal mean height of the young man and psychosis led us to conclude that patients with LFS may be those taller than the normal range. The results provided insights into clinical and therapeutic remedies and highlighted the need to carefully examine the psychological and neuropsychological symptoms of LFS.
\end{abstract}

Keywords: Lujan-Frynz Syndrome, Genetic, Mental Retardation, Psychosis

\section{Introduction}

Fryns indicated that the main clinical criteria for the diagnosis of Lujan-Fryns syndrome (LFS) include (a) mild to moderate mental retardation, (b) Marfanoid habitus, (c) general muscle relaxation and nasal twang, (d) normal secondary sexual development, and (e) typical craniofacial appearance with a prominent forehead, a long and narrow face, maxillary hypoplasia, a long nose with a high and narrow nasal bridge, a short and deep philtrum, a thin upper lip, and a deep palate (1). Although psychiatric disorders are not among the diagnostic criteria for LFS, most reported cases exhibit behavioral or psychiatric conditions (1), suggesting that LFS should be considered in the differential diagnosis of schizophrenia (1). The most common psychiatric illnesses and behavioral disorders associated with LFS are those related to autism spectrum disorders $(1,2)$. Other psychiatric conditions with behavioral manifestations observed in LFS patients include psychotic behavior (1), schizophrenia $(1,3)$, attention deficit hyperactivity disorder (ADHD) (3), extreme embarrassment (1,
3), obsessive-compulsive disorder (3), low frustration tolerance (4), learning disability (4), short-term memory impairment (4), social dysfunction (4), impaired momentum control (3), pyromania (1), and eating disorders and malnutrition associated with loss of appetite (5).

LFS patients need special educational services, psychological assessment, and a serious consideration for the early diagnosis of psychiatric disorders (6). Adults with LFS are tall, but their heights fall within the normal range (7). Specifically, their heights vary between the 10th and 90th percentiles and never exceed the 97th (7). No LFS patient with a height exceeding $97 \%$ has been reported in the medical literature published in English. The present research deviates from other studies in that it introduces a patient with a height above the 97th percentile, marfanoid characteristics, distinctive craniofacial appearance, and behavioral and mental disorders. The patient's excessive hyperactivity warranted monitoring by a neurologist during childhood, and the behavioral problems and excessive height growth that later developed necessitated monitoring by a pediatric psychiatrist and endocrinologist. How- 
ever, the patient was not diagnosed as suffering from LFS at the time. One of the essential requirements for a psychiatrist is to learn about diagnosing the psychiatric symptoms associated with LFS. This study recommends that LFS be considered in the differential diagnosis of mental disability and schizophrenia.

\section{Case Presentation}

The patient was an 18-year-old male who was referred to the psychosomatic clinic in an educational general hospital in Sari, Iran in November 2016 because of irritability, physical aggression, and self-harm with knife. He was subsequently admitted into the psychosomatic ward of the hospital, interviewed, and examined. According to the patient and his parents, his problems began to escalate about 4 months back, and visual and auditory hallucinations associated with the devil were expressed as the causes of his behavior. For example, he once attacked his father, choking him, presumably on the orders of the devil. On admission, he was well-informed; although he was cautious, he showed good cooperation in the interview. During the interview, he confirmed that he had seen Satan, who ordered him to harm himself and attack his father. He exhibited no suicidal thoughts but was excessively sentimental and given to non-abstract thinking. His substance history was negative. The patient had Marfanoid features. The clinical examination showed that he is tall with certain craniofacial features, such as acromegaly with a prominent forehead, a long and narrow face, a narrow and long nasal bridge, a small chin, maxillary hypoplasia, large ears, and long hands and fingers. He has hypernasal speech, pectus excavatum (Figure1), long marfanoid habitus with long limbs (Figure 2), and long flexing fingers. The oral examination revealed a deep palate and irregular teeth in two rows (Figure 3). His ophthalmic examination was normal, as were the findings of his pituitary magnetic resonance imaging exam. His testicles are slightly larger than normal. His height is $201 \mathrm{~cm}$ (The 97th percentile in men at this age is $190 \mathrm{~cm}$.), and his arm length is $207 \mathrm{~cm}$ (Figure 4). He has always been lean and showed a brief kyphosis. Given that his height exceeds the normal range, he was treated with testosterone seven months ago before admission. All routine examinations were performed (Table 1). Echocardiography shows mild to moderate mitral valve regurgitation and left ventricular hypertrophy with EF: 45\% - 50\%.

The mother had a normal pregnancy, and she and her husband have no history of alcohol abuse. The patient had been born after 9 months through a cesarean section. At birth, he had an Apgar score of 9 to 10, a weight of $4500 \mathrm{~g}$,

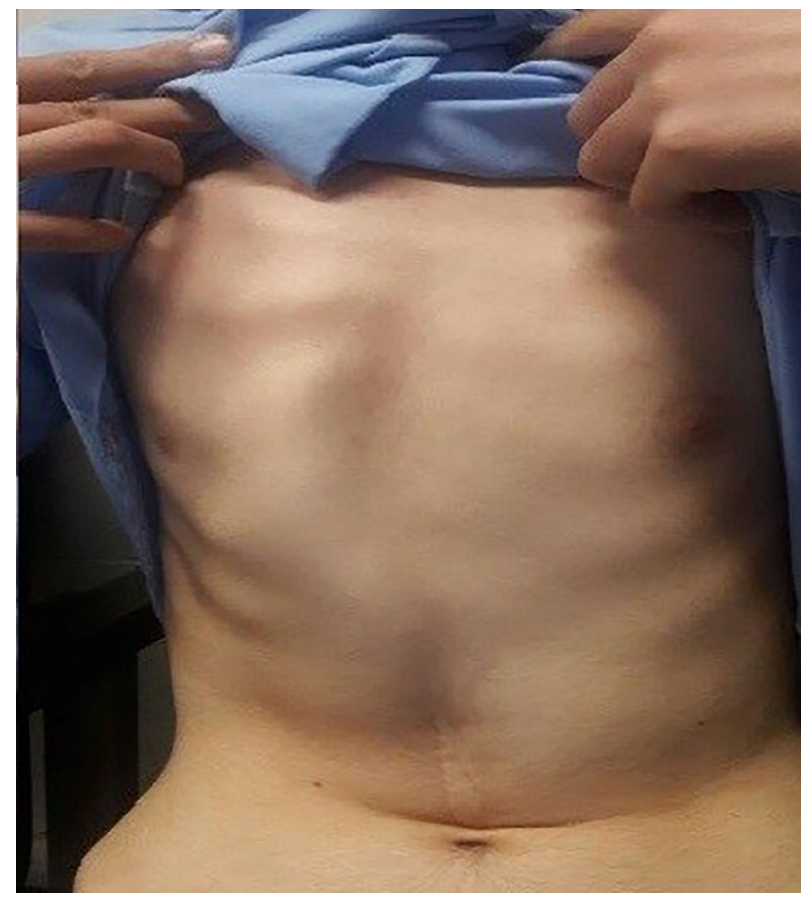

Figure 1. Pectus excavatum

a height of $52 \mathrm{~cm}$ (50th to 75th percentile), and a head circumference of $36 \mathrm{~cm}$ (90th percentile). The patient's prenatal period was normal, but his growth was delayed, and he has had a learning disability since childhood. By age 2.5 years, he was able to consume only fluids, and he underwent surgery to correct his reflux. After surgery, a cardiac examination was initiated because of the occurrence of tachycardia, ending with a diagnosis of mitral valve regurgitation. The condition was treated with enalapril. He showed high-risk behaviors and emotional instability in his childhood, with intermittent aggression and aggressive behavior worsening when he was in crowded places.

The patient suffers from moderate mental disability (at age 8 , his intelligence quotient (IQ) test score was 53). At the age of 8 , his pediatric neurologist started him on Ritalin to treat his aggression and learning difficulties. At nine years old, he was placed under the supervision of a psychiatrist because of aggression and incuriosity, and risperidone was added to his Ritalin regimen. Although Ritalin and risperidone controlled his symptoms, his behavioral disorders and aggression worsened after puberty (age 15). His nocturnal enuresis was significant.

After a full history was taken and physical, psychological, and behavioral profiles were established (with symptoms being Marfanoid features, tallness, specific craniofa- 


\begin{tabular}{|c|c|c|}
\hline Lab & Result & Normal Range \\
\hline TSH & $3.52 \mathrm{MIU} / \mathrm{L}$ & \\
\hline T4 & $10.55 \mu \mathrm{g} / \mathrm{dL}$ & \\
\hline T3 & $2.05 \mathrm{ng} / \mathrm{dL}$ & \\
\hline Vit D3-25-OHD & $16 \mathrm{ng} / \mathrm{dL}$ & Deficency $<20$ \\
\hline Prolactin & $0.7 \mathrm{ng} / \mathrm{dL}$ & \\
\hline IGF-1 & $203.2 \mathrm{ng} / \mathrm{dL}$ & $102-520$ \\
\hline GH basal & $0.12 \mathrm{ng} / \mathrm{dL}$ & $0.06-6.88$ \\
\hline LH & $3.6 \mathrm{MU} / \mathrm{mL}$ & \\
\hline FSH & $7.1 \mathrm{U} / \mathrm{mL}$ & \\
\hline Testosterone & $4.1 \mathrm{ng} / \mathrm{dL}$ & $2-9.8$ \\
\hline Serum homocysteine levels & $12.39 \mathrm{mmol} / \mathrm{L}$ & $5-15$ \\
\hline Echocardiography & Mild to moderate mitral valve regurgitation and left ventricular hypertrophy with EF: $45 \%$ - $50 \%$ & \\
\hline Gated spect myocardial perfusion scan & $\begin{array}{l}\text { Mild stress-induced ischemia is suggested in the apical anterolateral segment (small zoon), dilated LV } \\
\text { cavity ( } E D V=129 \mathrm{~mL} \text { ), estimated global } \mathrm{LV}=64 \% .\end{array}$ & \\
\hline Ophthalmologic evaluations & NL & \\
\hline
\end{tabular}

cial appearance, teeth in two rows, mental and behavioral disorders, and visual and auditory hallucinations), the possibility of a clinical syndrome was explored. The patient has a brother who is also tall, lean, and with learning disabilities. The possibility of an inherited X-linked disorder was therefore raised and LFS was the final diagnosis after a rule out of differential diagnoses.

A written informed consent was obtained from the patient's parents for the publication of this case report.

\section{Discussion}

The patient was evaluated as a typical case of LFS. As previously stated, no study prior to the current report has presented an LFS patient with a height exceeding the 97th percentile. Our patient has an abnormal height because his epiphyseal plate remains open, which suggests that LFS can be accompanied by a height higher than the normal range. It was diagnosed at the age of 18 with the help of a psychiatrist. He has been supervised by various doctors since childhood owing to numerous problems. The mean age of patients diagnosed with LFS is 25 years, as indicated in many published articles that we reviewed.

The clinical diagnostic criteria provided by Fryns are a tall height, Marfanoid features, long hands with highly flexible fingers, a nasal twang, muscle relaxation, mild to moderate mental retardation, behavioral disorders, and obvious facial deformity $(1,7)$. The craniofacial features of LFS patients include macrocephaly, a prominent forehead, a long and narrow face, maxillary hypoplasia, a high arched and narrow palate with uneven teeth, a weak chin, a long nose with a high and narrow nasal bridge, a short and deep philtrum, a thin upper lip, and low-set ears (8). In some cases, brain imaging showed agenesis of the corpus callosum (8). We found that a diagnosis of ADHD is associated with LFS cases reported in published psychiatric evaluations $(1,3)$. More than $90 \%$ of LFS cases are accompanied by a type of psychiatric disorder, such as aggressive behavior, pyromania, and alcohol consumption-related disorders, which may be related to personality disorders (1). The diseases often associated with LFS are autism spectrum disorders (62.5\% showed difficulty in establishing social relationships, tendency to isolate, and shyness), emotional instability (31.25\% showed low self-confidence, mood swings, irritability, recurrent concerns, feeling of losing control, aggressive behavior, suspicion, mistrust, etc.), and hyperactivity (18.75\%). Psychotic disorders (delusions and/or hallucinations) have been observed in $9.37 \%$ of LFS cases (1). De Hert et al. recommended that LFS be considered in the differential diagnosis of schizophrenia, as well as relevant behavioral problems (hyperactivity, aggressiveness, and pseudo-autistic behavior) (1). The high prevalence of psychiatric disorders in these patients requires psychiatric evaluation at diagnosis (1). The psychiatric problems of our case are consistent with his LFS, and he was diagnosed with psychosis because of other medical conditions (i.e., LFS).

Although LFS is usually taken into consideration when 


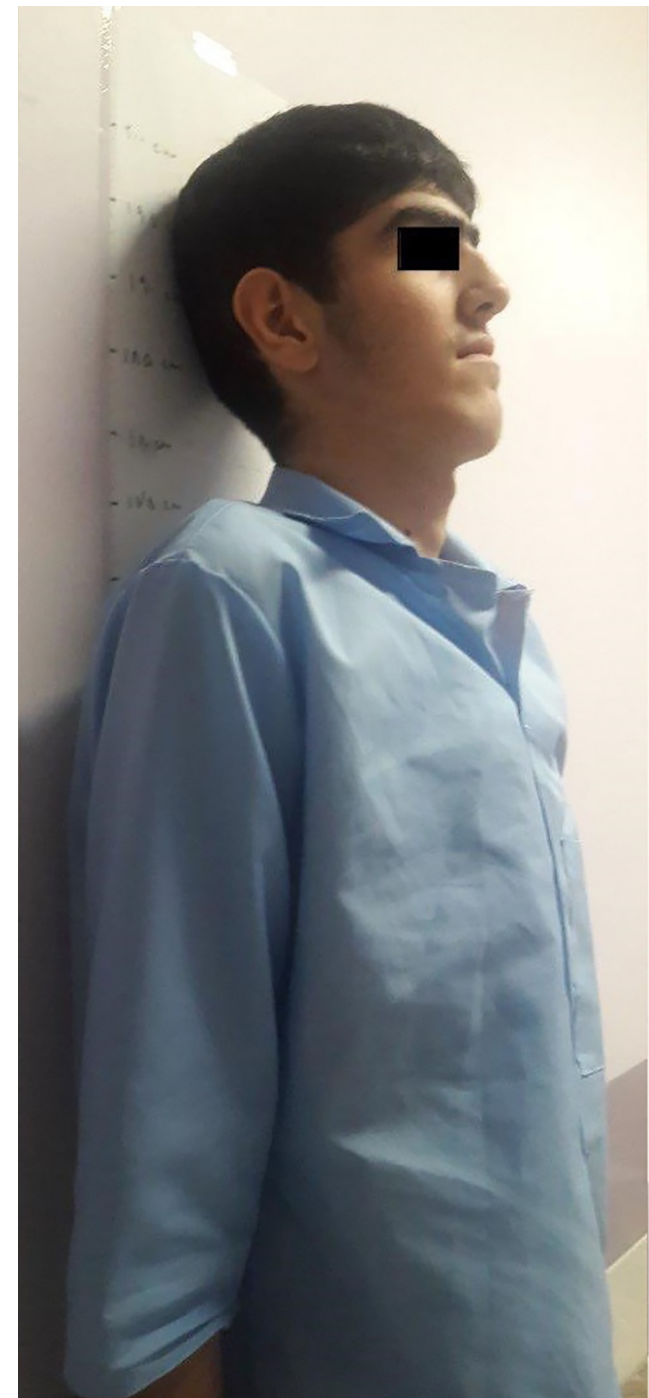

Figure 2. Tall marfanoid status

both mental disabilities and Marfanoid characteristics are diagnosed in one patient, LFS diagnosis is confirmed through the p.N1007S mutation in the MED12 gene (9). Opitz-Kaveggia is another syndrome that shows some of the characteristics and symptoms of LFS and is also related to mutation in the MED12 gene $(9,10)$. The diagnosis of LFS is based on clinical manifestations and the rejection of other disorders, including Marfan syndrome (7), LoeysDietz syndrome (1,11), Klinefelter syndrome (47,XXY), 47XYY syndrome, 22q11 deletion syndrome(Shprintzen syndrome or Velo-cardio-facial syndrome), Fragile X syndrome, and homocystinuria. No specific diagnostic test for verifying the diagnosis exists, but rejection should be followed by

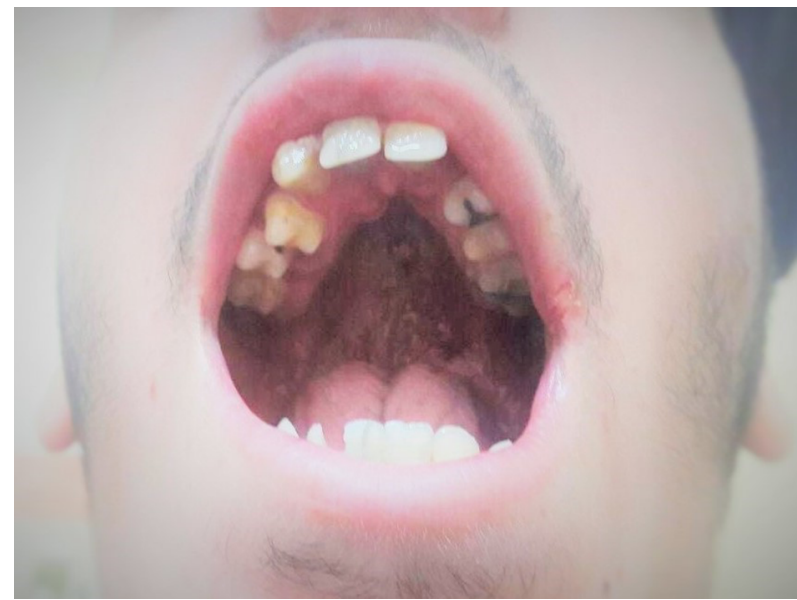

Figure 3. Deep palate and irregular teeth in two rows

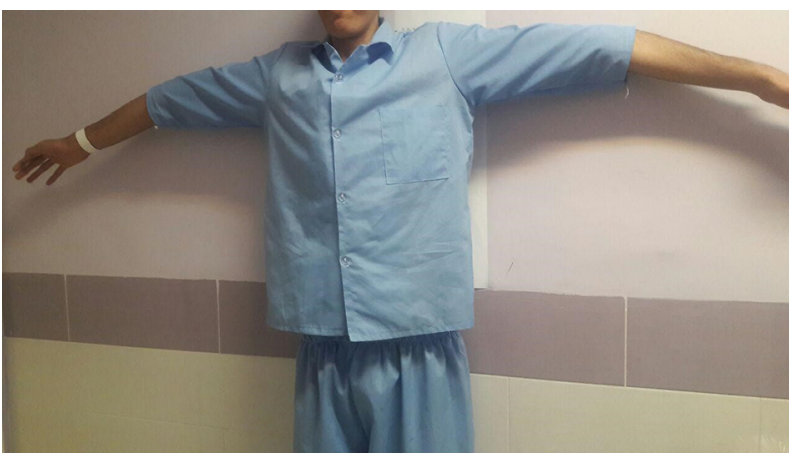

Figure 4. Long slender extremities

certain examinations, including echocardiography, cardiac and eye exams, determination of homocysteine levels in serum and urine, and chromosomal analysis. We rejected these differential diagnoses on the basis of the patient's clinical features and laboratory data. The parents could not afford to pay for chromosomal analysis, making this one of the weaknesses of this study.

No specific treatment for LFS has been developed, so patients should be monitored periodically to prevent the exacerbation of symptoms (7). In general, patients need special training and psychological support. Intensive attention and specialized care, including methods for assessing mental health and established therapies and educational programs should be provided to diagnose and prevent psychiatric disorders and related behavioral problems.

\subsection{Conclusions}

Lujan-Fryns syndrome is in the differential diagnosis of mental disorders and schizophrenia. The results provided 
insights into clinical and therapeutic remedies and highlighted the need to carefully examine the psychological and neuropsychological symptoms of LFS. Given that some patients may require specific rehabilitation programs, an essential strategy is to adopt a multi-disciplinary approach to LFS treatment.

\section{Acknowledgments}

We appreciate the patient's mother, who gave her information to us patiently.

\section{Footnotes}

Conflict of Interests: The authors declare that they had no competing interests.

Funding/Support: None.

Informed Consent: A written informed consent was obtained from the patient's parents for the publication of this case report.

\section{References}

1. Lerma-Carrillo I, Molina JD, Cuevas-Duran T, Julve-Correcher C, EspejoSaavedra JM, Andrade-Rosa C, et al. Psychopathology in the LujanFryns syndrome: Report of two patients and review. Am J Med Genet A. 2006;140(24):2807-11. doi: 10.1002/ajmg.a.31503. [PubMed: 17036352].

2. Artigas-Pallares J, Gabau-Vila E, Guitart-Feliubadalo M. [Syndromic autism: II. Genetic syndromes associated with autism]. Rev Neurol. 2005;40 Suppl 1:S151-62. Spanish. [PubMed: 15736079].
3. Purandare KN, Markar TN. Psychiatric symptomatology of LujanFryns syndrome: An X-linked syndrome displaying Marfanoid symptoms with autistic features, hyperactivity, shyness and schizophreniform symptoms. Psychiatr Genet. 2005;15(3):229-31. doi: 10.1097/00041444-200509000-00016. [PubMed: 16094260].

4. Williams MS. Neuropsychological evaluation in Lujan-Fryns syndrome: Commentary and clinical report. Am J Med Genet A. 2006;140(24):2812-5. doi: 10.1002/ajmg.a.31501. [PubMed: 17103446].

5. Alonso P, Pintos G, Almazan F, Hernandez L, Loran E, Menchon $\mathrm{JM}$, et al. Eating disorder in a patient with phenotypical features of Lujan-Fryns syndrome. Clin Dysmorphol. 2006;15(3):181-4. doi: 10.1097/01.mcd.0000220610.24908.a4. [PubMed:16760741].

6. Basu S, Patil B, Aundhakar CD, Ingale SY. A rare case of Lujan fryn syndrome. Int J Recent Trends Sci Tech. 2014;9(3):341-2.

7. Van Buggenhout G, Fryns JP. Lujan-Fryns syndrome (mental retardation, X-linked, marfanoid habitus). Orphanet J Rare Dis. 2006;1:26. doi: 10.1186/1750-1172-1-26. [PubMed: 16831221]. [PubMed Central: PMC1538574].

8. Dieckmann PM, Lucena LC, Dutra LA, Pedroso JL, Barsottini OG. Marfanoid features and X-linked mental retardation associated with craniofacial abnormalities: The Lujan-Fryns syndrome. Arq Neuropsiquiatr. 2013;71(1):68-9. doi: 10.1590/s0004-282x2013000100017. [PubMed: 23338167].

9. Schwartz CE, Tarpey PS, Lubs HA, Verloes A, May MM, Risheg H, et al. The original Lujan syndrome family has a novel missense mutation (p.N1007S) in the MED12 gene. J Med Genet. 2007;44(7):472-7. doi: 10.1136/jmg.2006.048637. [PubMed: 17369503]. [PubMed Central: PMC2597996].

10. Charzewska A, Maiwald R, Kahrizi K, Oehl-Jaschkowitz B, Dufke A, Lemke JR, et al. The power of the Mediator complex-expanding the genetic architecture and phenotypic spectrum of MED12-related disorders. Clin Genet. 2018;94(5):450-6. doi: 10.1111/cge.13412. [PubMed: 30006928].

11. Murphy-Ryan M, Psychogios A, Lindor NM. Hereditary disorders of connective tissue: a guide to the emerging differential diagnosis. Genet Med. 2010;12(6):344-54. doi: 10.1097/GIM.0b013e3181e074fo. [PubMed: 20467323]. 